\title{
Vibration of thick plates using finite strip-elements
}

\author{
J. Petrolito* $\quad$ B.W. Golley ${ }^{\dagger}$
}

(Received 7 August 2000)

\begin{abstract}
This paper develops a finite strip-element method for the vibration analysis of thick plates. The method uses a combined polynomial and trigonometric interpolation scheme that enables all boundary conditions to be correctly treated. The global equations are derived in the

*Division of Physical Sciences and Engineering, La Trobe University, Bendigo, Victoria, Australia. mailto:j.petrolito@bendigo.latrobe.edu.au

${ }^{\dagger}$ School of Civil Engineering, Australian Defence Force Academy, Canberra,

${ }^{0}$ See http://anziamj . austms.org. au/V42/CTAC99/Petr for this article and ancillary services, (C) Austral. Mathematical Soc. 2000. Published 27 Nov 2000.
\end{abstract} Australia. 
usual manner of the finite element method, and natural frequencies of vibration can be found by solving a linear eigenvalue problem.

\section{Contents}

1 Introduction

C1139

2 Governing Equations

C1141

3 Strip-Element Interpolation

C1144

4 Formulation of Strip-Element Matrices

C1148

5 Examples

C1151

5.1 Example

C1151

5.2 Example 2

C1153

6 Conclusions

C1153

References

C1154 


\section{Introduction}

The efficient analysis of plate structures is of fundamental importance in many branches of engineering, and continues to be an area of active research. While the governing differential equations for particular models of plate analysis are well-established, it is generally not possible to obtain analytical solutions of the equations except for simple geometries. Hence, numerical solutions are required for practical problems. By far the most popular method in use for plate analysis is the finite element method [1]. Its strength lies in its generality and its ability to easily deal with complex geometries and loading conditions.

However, the full generality of the method is not required when the geometry of the problem is regular, as is frequently the case for many practical structures, for example bridges and buildings. In such cases, the efficiency of the analysis can be improved by adopting alternative approximation schemes that explicitly account for the regularity of the structure. One technique that is suitable in such cases is the finite strip method.

Finite strips have now been used for the analysis of plates for some thirty years [2]. The method was initially developed for analysing thin rectangular plates with two opposite edges simply supported [3]. In this case, the global equations uncouple into a number of smaller systems of equations owing to certain orthogonality relationships resulting from the chosen displacement functions. This leads to a reduction in storage requirements for the global 
equations and an increase in computational efficiency when compared with the finite element method. Later work enabled other boundary conditions to be treated. However, in all other cases uncoupling of the equations does not occur, and the method loses some of its efficiency. A more serious problem is that the shape functions used in the finite strip method do not satisfy free edge boundary conditions, which frequently arise in practice.

To overcome these problems, while retaining the basic features of the finite strip method, the authors developed a method that combined the interpolation schemes of finite elements and finite strips. This method, called the finite strip-element method, has been used by the authors and their colleagues for a variety of problems, for example [4, 5, 6, 7]. A key advantage of the finite strip-element method is that the method correctly treats all boundary conditions.

In this paper, we extend the finite strip-element method to the dynamic analysis of thick plate structures. The use of a thick plate theory allows a wide range of plate structures to be analysed, and the theory is also applicable to thin plate structures. By using combined polynomial and trigonometric approximations, the formulation allows all boundary conditions to be handled in a simple manner. Moreover, the formulation leads to positive definite stiffness and mass matrices, and enables standard eigenvalue routines to be used for calculating natural frequencies of vibration. 


\section{Governing Equations}

The influence of shear deformation is critical for thick plate problems, and there are various theories available to account for this effect. The simplest and most-used theory is Mindlin's theory [8]. The theory is applicable to both thick and thin plates, and it incorporates independent assumptions for the transverse displacement $w$ and normal rotations $\theta_{x}$ and $\theta_{y}$ (see Figure 1 ). This allows $C_{0}$ approximations to be used for these variables.

In contrast to thin plate theory, both bending and transverse shear strains are present. These are given by

$$
\boldsymbol{\epsilon}_{b}=\left[\begin{array}{cc}
\partial / \partial x & 0 \\
0 & \partial / \partial y \\
\partial / \partial y & \partial / \partial x
\end{array}\right]\left\{\begin{array}{c}
\theta_{x} \\
\theta_{y}
\end{array}\right\}=\mathbf{L}_{1} \boldsymbol{\theta}, \quad \boldsymbol{\epsilon}_{s}=\left\{\begin{array}{c}
\partial / \partial x \\
\partial / \partial y
\end{array}\right\} w-\boldsymbol{\theta}=\mathbf{L}_{2} w-\boldsymbol{\theta}
$$

where $\boldsymbol{\epsilon}_{b}$ is the bending strain vector and $\boldsymbol{\epsilon}_{s}$ is the shear strain vector, which is zero in the thin plate limit.

For an isotropic material, bending moments and shears are given in terms of the strains by

$\mathbf{M}=\left\{\begin{array}{c}M_{x} \\ M_{y} \\ M_{x y}\end{array}\right\}=-D\left[\begin{array}{ccc}1 & \nu & 0 \\ \nu & 1 & 0 \\ 0 & 0 & (1-\nu) / 2\end{array}\right] \boldsymbol{\epsilon}_{b}=-\mathbf{D} \boldsymbol{\epsilon}_{b}, \mathbf{Q}=\left\{\begin{array}{l}Q_{x} \\ Q_{y}\end{array}\right\}=k G h \boldsymbol{\epsilon}_{s}$ 


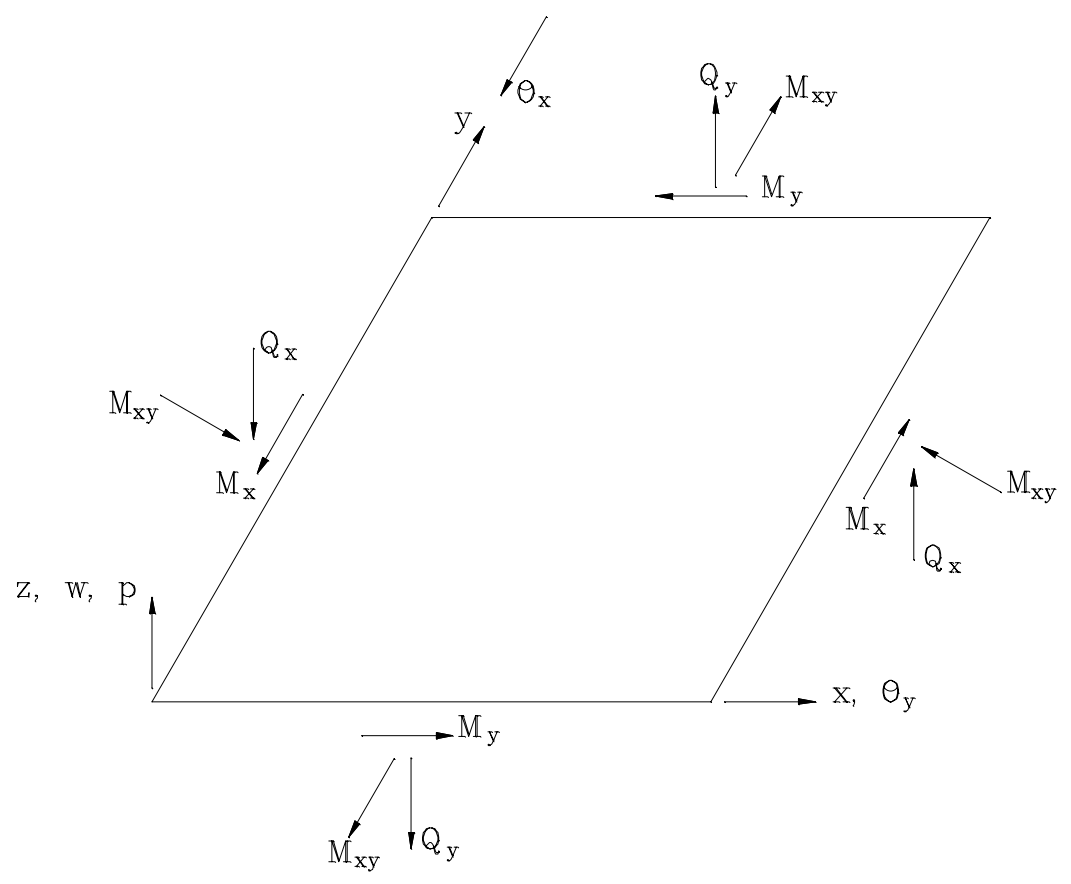

Figure 1: Sign convention. 
In equation (2), $D=E h^{3} /\left(1-\nu^{2}\right)$ is the bending rigidity, $E$ is Young's modulus, $\nu$ is Poisson's ratio, $G=E / 2(1+\nu)$ is the shear modulus, $h$ is the plate thickness and $k$ is a correction factor, which is taken as $\pi^{2} / 12$ for dynamics problems.

The dynamic equilibrium equations for Mindlin's theory are

$$
\mathbf{Q}-\mathbf{L}_{1}^{T} \mathbf{M}=\frac{\rho h^{3}}{12} \ddot{\boldsymbol{\theta}}, \quad \mathbf{L}_{2}^{T} \mathbf{Q}+p=\rho h \ddot{w}
$$

where $\rho$ is the material density, $p$ is the applied transverse load on the plate and a dot denotes partial differentiation with respect to time, $t$.

Combining equations (1)-(3) gives the governing equations, namely

$$
\begin{aligned}
D\left[\theta_{x, x x}+\frac{(1-\nu)}{2} \theta_{x, y y}+\frac{(1+\nu)}{2} \theta_{y, x y}\right]+k G h\left(w_{, x}-\theta_{x}\right) & =\frac{\rho h^{3}}{12} \ddot{\theta}_{x} \\
D\left[\theta_{y, y y}+\frac{(1-\nu)}{2} \theta_{y, x x}+\frac{(1+\nu)}{2} \theta_{x, x y}\right]+k G h\left(w_{, y}-\theta_{y}\right) & =\frac{\rho h^{3}}{12} \ddot{\theta}_{y} \\
k G h\left(w_{, x x}-\theta_{x, x}+w_{, y y}-\theta_{y, y}\right)+p & =\rho h \ddot{w}
\end{aligned}
$$

where subscripts after the comma denote partial differentiation. Equation (4) represents a system of three coupled equations of order six, and requires the specification of three boundary conditions on any portion of the plate boundary.

If the applied load varies sinusoidally with time, such that

$$
p=p(x, y, t)=\bar{p}(x, y) \sin \Omega t
$$


where $\Omega$ is the frequency of the applied load, we have

$$
\begin{aligned}
w & =w(x, y, t)=\bar{w}(x, y) \sin \Omega t \\
\theta_{x}=\theta_{x}(x, y, t) & =\overline{\theta_{x}}(x, y) \sin \Omega t \\
\theta_{y} & =\theta_{y}(x, y, t)=\overline{\theta_{y}}(x, y) \sin \Omega t
\end{aligned}
$$

Substituting equations (5) and (6) into equation (4) gives

$$
\begin{aligned}
D\left[\bar{\theta}_{x, x x}+\frac{(1-\nu)}{2} \bar{\theta}_{x, y y}+\frac{(1+\nu)}{2} \bar{\theta}_{y, x y}\right]+k G h\left(\bar{w}_{, x}-\bar{\theta}_{x}\right) & =-\frac{\rho h^{3} \Omega^{2}}{12} \bar{\theta}_{x} \\
D\left[\bar{\theta}_{y, y y}+\frac{(1-\nu)}{2} \bar{\theta}_{y, x x}+\frac{(1+\nu)}{2} \bar{\theta}_{y, x y}\right]+k G h\left(\bar{w}_{, y}-\bar{\theta}_{y}\right) & =-\frac{\rho h^{3} \Omega^{2}}{12} \bar{\theta}_{y}(7) \\
k G h\left(\bar{w}_{, x x}-\bar{\theta}_{x, x}+\bar{w}_{, y y}-\bar{\theta}_{y, y}\right)+\bar{p} & =-\rho h \Omega^{2} \bar{w}
\end{aligned}
$$

Equation (7) is in terms of amplitudes only, and thus only amplitudes of relevant quantities are considered in the remainder of the paper. In addition, the bars on all quantities are dropped for convenience.

\section{Strip-Element Interpolation}

Figure 2 shows a typical plate structure that is suitable for analysis using the proposed method. The structure has been subdivided into $J$ strip-elements of width $b^{j}$, which have $L$ equidistant nodal lines running in the $x$ direction. 


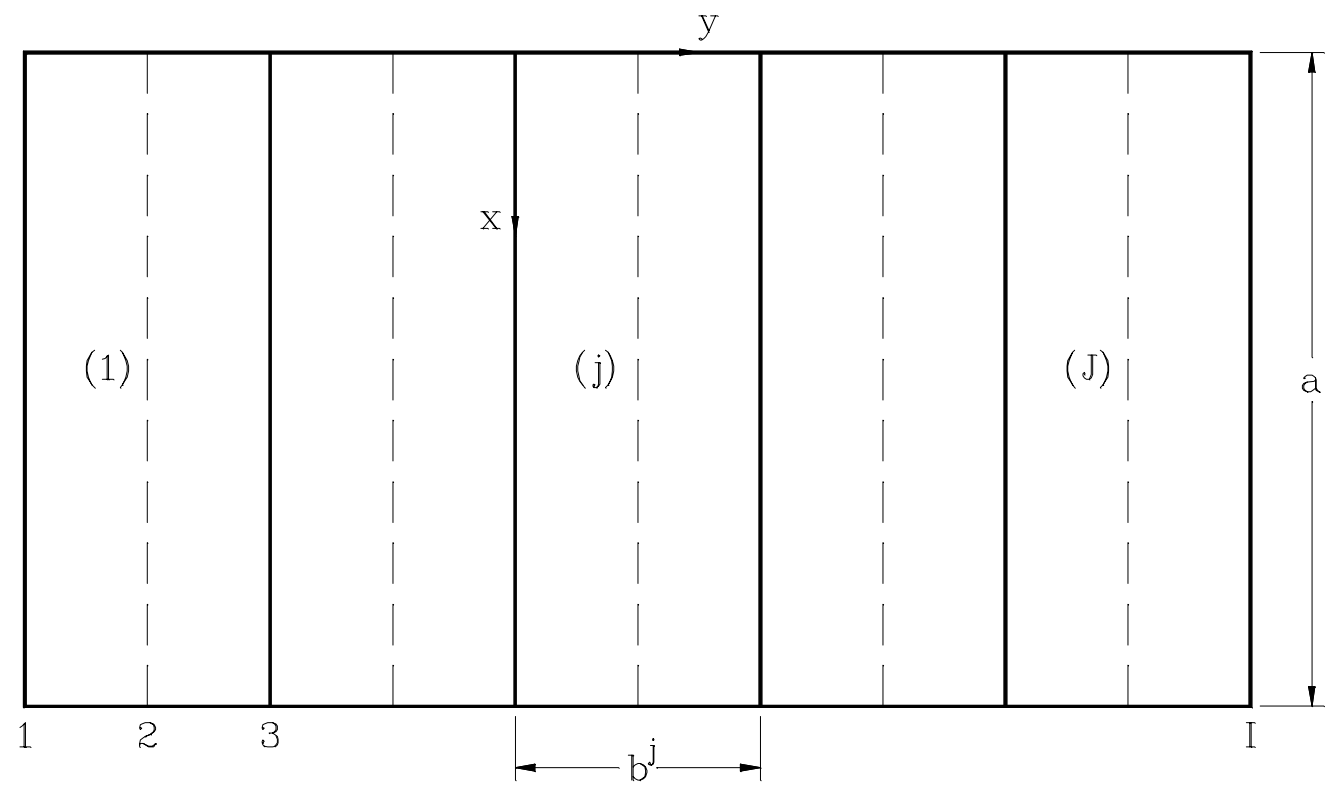

FiguRE 2: Typical structure suitable for analysis. 
There are $I=J(L-1)+1$ nodal lines in the structure. Different boundary conditions can be applied at the ends $x=0$ and $x=a$ of the strip-element.

On nodal line $i$, which is the $l$ th nodal line of the $j$ th strip-element, continuity of displacements and rotations is ensured by taking

$$
w^{i}(\xi) \equiv w^{j, l}(\xi), \quad \theta_{x}^{i}(\xi) \equiv \theta_{x}^{j, l}(\xi), \quad \theta_{y}^{i}(\xi) \equiv \theta_{y}^{j, l}(\xi)
$$

where $\xi=x / a$ and $a$ is the strip-element length.

Within strip-element $j$, the displacement and rotations are approximated by

$$
\begin{aligned}
w^{j}(x, y) & =\sum_{l=1}^{L} w^{j, l}(\xi) f_{l}^{L}(\eta) \\
\theta_{x}^{j}(x, y) & =\sum_{l=1}^{L} \theta_{x}^{j, l}(\xi) f_{l}^{L}(\eta) \\
\theta_{y}^{j}(x, y) & =\sum_{l=1}^{L} \theta_{y}^{j, l}(\xi) f_{l}^{L}(\eta)
\end{aligned}
$$

where $\eta=y / b^{j}$, and the functions $f_{l}^{L}(\eta)$ are Lagrange polynomials defined as follows for $L=2,3$ and 4 :

$$
\begin{array}{lll}
f_{1}^{2}(\xi)=1-\xi, & f_{1}^{3}(\xi)=1-3 \xi+2 \xi^{2}, & f_{1}^{4}(\xi)=\frac{1}{2}\left(2-11 \xi+18 \xi^{2}-9 \xi^{3}\right) \\
f_{2}^{2}(\xi)=\xi, & f_{2}^{3}(\xi)=4 \xi-4 \xi^{2}, & f_{2}^{4}(\xi)=\frac{9}{2}\left(2 \xi-5 \xi^{2}+3 \xi^{3}\right) \\
& f_{3}^{3}(\xi)=-\xi+2 \xi^{2}, & f_{3}^{4}(\xi)=\frac{9}{2}\left(-\xi+4 \xi^{2}-3 \xi^{3}\right) \\
& f_{4}^{4}(\xi)=\frac{1}{2}\left(2 \xi-9 \xi^{2}+9 \xi^{3}\right)
\end{array}
$$


The approximations in equation (9) with $L \leq 4$ allow strip-elements with up to a cubic order approximation in the $y$ direction to be developed. The examples considered below will demonstrate that this is sufficient in practice. However, strip-elements with higher order approximations can be developed in an analogous manner by simply increasing the value of $L$, and this procedure is independent of any specified boundary conditions.

Along a nodal line, the displacements and rotations are approximated by the sum of polynomial and trigonometric terms. Different forms of this approximation can be used depending on the nature of the analysis and the method that is used to enforce any required boundary conditions at the strip-element ends [5].

For dynamics problems, it is computationally convenient to use a modified approximation scheme that leads to a positive-definite stiffness matrix. Hence, the displacement and rotations along nodal line $i$ are approximated by

$$
\begin{aligned}
w^{i}(\xi)= & \sum_{m=1}^{M} W_{m}^{j, l} \sin \alpha_{m} \xi+\widetilde{W}_{1}^{j, l} f_{1}^{3}(\xi)+\widetilde{W}_{2}^{j, l} f_{2}^{3}(\xi)+\widetilde{W}_{3}^{j, l} f_{3}^{3}(\xi) \\
\theta_{x}^{i}(\xi)= & \sum_{m=1}^{M} \Theta_{x m}^{j, l}\left(\cos \alpha_{m} \xi-f_{1}^{3}(\xi)-(-1)^{m} f_{3}^{3}(\xi)\right) \\
& +\widetilde{\Theta}_{x 1}^{j, l} f_{1}^{3}(\xi)+\widetilde{\Theta}_{x 2}^{j, l} f_{2}^{3}(\xi)+\widetilde{\Theta}_{x 3}^{j, l} f_{3}^{3}(\xi) \\
\theta_{y}^{i}(\xi)= & \sum_{m=1}^{M} \Theta_{y m}^{j, l} \sin \alpha_{m} \xi+\widetilde{\Theta}_{y 1}^{j, l} f_{1}^{3}(\xi)+\widetilde{\Theta}_{y 2}^{j, l} f_{2}^{3}(\xi)+\widetilde{\Theta}_{y 3}^{j, l} f_{3}^{3}(\xi)
\end{aligned}
$$


where $\alpha_{m}=m \pi$ and $M$ is the total number of harmonics. The coefficients $W_{m}^{j, l}, \widetilde{W}_{1}^{j, l}, \widetilde{W}_{2}^{j, l}, \ldots, \widetilde{\Theta}_{y 3}^{j, l}$ are in general to be determined. Any boundary conditions are simply treated by specifying appropriate values of some of these coefficients.

Equation (10) can alternatively be written in matrix form as

$$
w^{j}(x, y)=\mathbf{N}_{w}^{j}(x, y) \mathbf{W}^{j}, \quad \boldsymbol{\theta}^{j}=\mathbf{N}_{\theta}^{j}(x, y) \boldsymbol{\Theta}^{j}
$$

where $\mathbf{N}_{w}$ and $\mathbf{N}_{\theta}$ are shape function vectors and $\mathbf{W}^{j}$ and $\boldsymbol{\Theta}^{j}$ are vectors of the strip-element coefficients.

\section{Formulation of Strip-Element Matrices}

For dynamics problems, we require the stiffness and mass matrices for the strip-element. From equations (1) and (11), the strains within the stripelement are obtained as

$$
\boldsymbol{\epsilon}_{b}^{j}=\mathbf{L}_{1} \mathbf{N}_{\theta}^{j} \boldsymbol{\Theta}^{j}=\mathbf{B}_{b}^{j} \boldsymbol{\Theta}^{j}, \quad \boldsymbol{\epsilon}_{s}^{j}=\mathbf{L}_{2} \mathbf{N}_{w} \mathbf{W}^{j}-\mathbf{N}_{\theta}^{j} \boldsymbol{\Theta}^{j}=\mathbf{B}_{s}^{j} \mathbf{W}^{j}-\mathbf{N}_{\theta}^{j} \boldsymbol{\Theta}^{j}
$$

The strip-element stiffness matrix $\mathbf{k}^{j}$ is obtained from its strain energy, giving

$$
\mathbf{k}^{j}=\int_{0}^{b^{j}} \int_{0}^{a}\left\{\left[\begin{array}{cc}
\mathbf{0} & \mathbf{B}_{b}^{j} \\
\mathbf{B}_{s}^{j} & -\mathbf{N}_{\theta}^{j}
\end{array}\right]^{T}\left[\begin{array}{cc}
\mathbf{D} & \mathbf{0} \\
\mathbf{0} & k G h \mathbf{I}
\end{array}\right]\left[\begin{array}{cc}
\mathbf{0} & \mathbf{B}_{b}^{j} \\
\mathbf{B}_{s}^{j} & -\mathbf{N}_{\theta}^{j}
\end{array}\right]\right\} d x d y
$$


where $\mathbf{I}$ is an identity matrix and $\mathbf{0}$ is a zero matrix.

Some care is required in performing the integrals in equation (13) to ensure that locking does not occur in the thin plate limit [5]. The integrals in equation (13) associated with the bending strain energy are performed analytically. However, for the integrals associated with the shear strain energy, integration in the $x$ direction is performed analytically whereas integration in the $y$ direction is performed using Gauss quadrature. The number of Gauss points, $G$, used is given by

$$
G=L-1
$$

In all cases this number is one less than that required to integrate the terms associated with the shear terms exactly in the $y$ direction.

The strip-element mass matrix $\mathbf{m}^{j}$ is obtained from its kinetic energy, giving

$$
\mathbf{m}^{j}=\rho h \int_{0}^{b^{j}} \int_{0}^{a}\left\{\left[\begin{array}{ll}
\left(\mathbf{N}_{w}\right)^{T} & \left(\mathbf{N}_{\theta}\right)^{T}
\end{array}\right]\left[\begin{array}{cc}
\mathbf{I} & \mathbf{0} \\
\mathbf{0} & \frac{h^{2}}{12} \mathbf{I}
\end{array}\right]\left[\begin{array}{l}
\mathbf{N}_{w} \\
\mathbf{N}_{\theta}
\end{array}\right]\right\} d x d y
$$

In contrast to the stiffness matrix, all the integrals in equation (15) are performed analytically.

The assembly of the global equations from the strip-element matrices follows standard finite element procedures [1]. The calculation of natural frequencies of vibration leads to a linear eigenvalue problem, namely

$$
\mathbf{K} \phi=\Omega^{2} \mathbf{M} \phi
$$


where $\mathbf{K}$ and $\mathbf{M}$ are the stiffness and mass matrices for the structure and $\phi$ is the total vector of unknown coefficients.

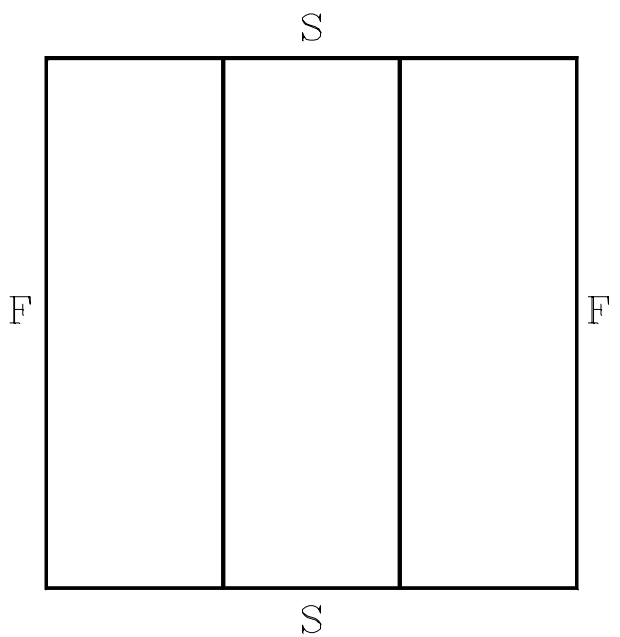

(a)

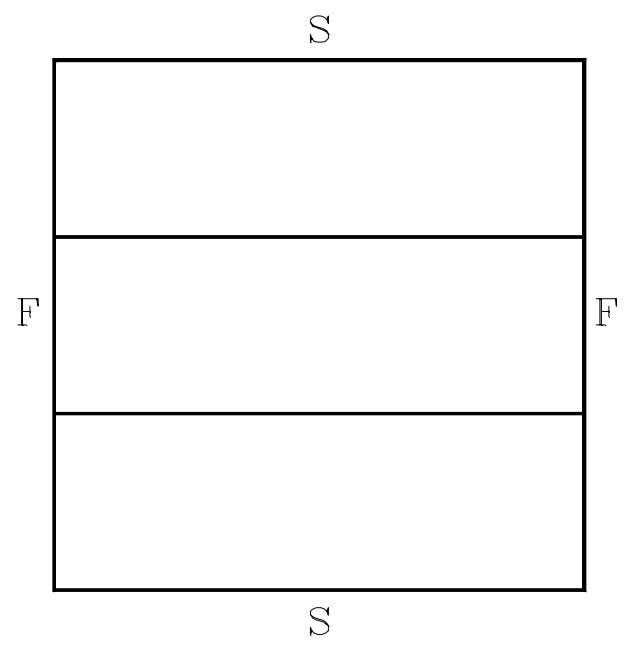

(b)

FIGURE 3: Example 1 showing alternate discretisations ( $\mathrm{S}=$ simple support, $\mathrm{F}=$ free edge). 


\section{Examples}

Two examples are considered to demonstrate the accuracy of the method. In both cases, Poisson's ratio was taken as 0.3 , and the results are given in terms of a frequency parameter, $\lambda$, defined as

$$
\lambda=\Omega a^{2} \sqrt{2(1+\nu) \rho / E}
$$

where $a$ is a reference length.

\section{$5.1 \quad$ Example 1}

As a first example, we consider the free vibration of a square plate of size $a \times a$ that is simply supported (S) on two opposite edges and free (F) on the other two edges (see Figure 3). The first natural frequency parameter is given in Tables 1-3 for the two alternate discretisations shown. The simple support condition is exactly satisfied for discretisation (a), and hence this discretisation leads to more accurate results for all cases. The results also show that a quadratic strip-element is significantly more accurate that a linear strip-element. However, the difference in accuracy between the quadratic and cubic strip-elements is not as great. It is also clear from the results that the method correctly treats the free edge boundary condition, and that accurate results are obtained with the solution of few equations. 
TABLE 1: Frequency parameter $\lambda_{1}$ for a square SFSF plate using linear stripelement $(a / h=10$, reference value $[9]=0.4606)$.

\begin{tabular}{|c|c|c|c|c|c|c|c|c|}
\hline \multirow{3}{*}{$M$} & \multicolumn{4}{|c|}{ Discretisation (a) } & \multicolumn{4}{c|}{ Discretisation (b) } \\
\cline { 2 - 9 } & $L=3$ & $L=5$ & $L=9$ & $L=17$ & $L=3$ & $L=5$ & $L=9$ & $L=17$ \\
\hline 0 & 0.5044 & 0.5116 & 0.5121 & 0.5121 & 0.6478 & 0.5007 & 0.4781 & 0.4650 \\
1 & 0.4525 & 0.4601 & 0.4606 & 0.4606 & 0.6457 & 0.4989 & 0.4701 & 0.4633 \\
3 & 0.4525 & 0.4601 & 0.4606 & 0.4606 & 0.6454 & 0.4986 & 0.4698 & 0.4630 \\
5 & 0.4525 & 0.4601 & 0.4606 & 0.4606 & 0.6453 & 0.4984 & 0.4697 & 0.4629 \\
\hline
\end{tabular}

TABLE 2: Frequency parameter $\lambda_{1}$ for a square SFSF plate using quadratic strip-element $(a / h=10$, reference value $[9]=0.4606)$.

\begin{tabular}{|c|c|c|c|c|c|c|c|c|}
\hline \multirow{3}{*}{$M$} & \multicolumn{4}{|c|}{ Discretisation (a) } & \multicolumn{4}{c|}{ Discretisation (b) } \\
\cline { 2 - 9 } & $L=3$ & $L=5$ & $L=9$ & $L=17$ & $L=3$ & $L=5$ & $L=9$ & $L=17$ \\
\hline 0 & 0.5030 & 0.5112 & 0.5121 & 0.5121 & 0.5141 & 0.4681 & 0.4631 & 0.4628 \\
1 & 0.4510 & 0.4597 & 0.4606 & 0.4606 & 0.5125 & 0.4663 & 0.4614 & 0.4610 \\
3 & 0.4510 & 0.4597 & 0.4606 & 0.4606 & 0.5122 & 0.4660 & 0.4611 & 0.4607 \\
5 & 0.4510 & 0.4597 & 0.4606 & 0.4606 & 0.5121 & 0.4659 & 0.4610 & 0.4606 \\
\hline
\end{tabular}


TABLE 3: Frequency parameter $\lambda_{1}$ for a square SFSF plate using cubic stripelement $(a / h=10$, reference value $[9]=0.4606)$.

\begin{tabular}{|c|c|c|c|c|c|c|c|c|}
\hline \multirow{2}{*}{$M$} & \multicolumn{4}{|c|}{ Discretisation (a) } & \multicolumn{4}{c|}{ Discretisation (b) } \\
\cline { 2 - 9 } & $L=4$ & $L=7$ & $L=10$ & $L=13$ & $L=4$ & $L=7$ & $L=10$ & $L=13$ \\
\hline 0 & 0.5128 & 0.5119 & 0.5121 & 0.5121 & 0.4688 & 0.4629 & 0.4628 & 0.4628 \\
1 & 0.4614 & 0.4604 & 0.4606 & 0.4606 & 0.4670 & 0.4611 & 0.4610 & 0.4610 \\
3 & 0.4614 & 0.4604 & 0.4606 & 0.4606 & 0.4667 & 0.4608 & 0.4607 & 0.4607 \\
5 & 0.4614 & 0.4604 & 0.4606 & 0.4606 & 0.4666 & 0.4607 & 0.4606 & 0.4606 \\
\hline
\end{tabular}

\subsection{Example 2}

Secondly, we consider the free vibration of the plate with all edges simply supported. To directly compare the different strip-elements, a quarter of the plate was considered with 7 and 13 nodal lines. The first frequency parameter is given in Table 4 for the two cases. Again, the results show that the quadratic strip-element is superior to the linear strip-element whereas these is little difference between the quadratic and cubic strip-elements.

\section{Conclusions}

This paper has presented a finite strip-element method for the dynamic analysis of thick plates. The method uses combined polynomial and trigonomet- 
TABLE 4: First frequency parameter $\lambda_{1}$ for simply supported square plate $(a / h=10$, exact value $=0.9300)$.

\begin{tabular}{|c|c|c|c|c|c|c|}
\hline \multirow{4}{*}{$M$} & \multicolumn{6}{|c|}{ Number of nodal lines } \\
\cline { 2 - 7 } & \multicolumn{3}{|c|}{$L=7$} & \multicolumn{3}{c|}{$L=13$} \\
\cline { 2 - 7 } & Linear & Quadratic & Cubic & Linear & Quadratic & Cubic \\
\hline 0 & 0.9501 & 0.9469 & 0.9468 & 0.9476 & 0.9468 & 0.9468 \\
1 & 0.9491 & 0.9459 & 0.9459 & 0.9467 & 0.9459 & 0.9459 \\
3 & 0.9351 & 0.9319 & 0.9318 & 0.9326 & 0.9318 & 0.9318 \\
5 & 0.9338 & 0.9305 & 0.9305 & 0.9313 & 0.9305 & 0.9305 \\
10 & 0.9334 & 0.9301 & 0.9300 & 0.9308 & 0.9300 & 0.9300 \\
\hline
\end{tabular}

ric approximations within each strip-element. The formulation of the global equations follows standard finite element procedures, and all boundary conditions are correctly treated by the method. The examples demonstrated that accurate solutions are obtained with the solution of few equations.

\section{References}

[1] O.C. Zienkiewicz and R.L. Taylor. The Finite Element Method, 4th edn, volumes 1 and 2. McGraw Hill, 1991, 1994.

[2] Y.K. Cheung and L.G. Tham. Finite Strip Method. CRC Press, 1998. 
[3] Y.K. Cheung. Finite strip method in the analysis of elastic plates with two opposite simply supported ends. Proc. Inst. Civ. Engrs., 40:1-7, 1968.

[4] B.W. Golley, W.A. Grice, and J. Petrolito. Plate bending analysis using finite strip-elements. J. Struct. Engrg., ASCE, 113:1282-1296, 1987.

[5] J. Petrolito, W.A. Grice, and B.W. Golley. Finite strip-elements for thick plate analysis. J. Struct. Engrg., ASCE, 115:1163-1182, 1989.

[6] B.W. Golley and W.A. Grice. Prismatic folded plate analysis using finite strip-elements. Computer Methods in Applied Mechanics and Engineering, 76:101-118, 1989.

[7] B.W. Golley and C. Downing. Dynamic analysis of cylindrical shells using finite strip-elements. Proc. Int. Conf. on Computational Methods in Engineering, pages 369-374, Singapore, 1992.

[8] R.D. Mindlin. Influence of rotatory inertia and shear on flexural motion of isotropic plates. J. Appl. Mech., 23:31-38, 1951.

[9] K.M. Liew, C.M. Wang, Y. Xiang, and S. Kitipornchai. Vibration of Mindlin Plates. Elsevier, 1998.

C1139, C1149 C1139 C1139 C1140 C1140, C1147, C1149 C1140 C1140 C1141 C1152, C1152, C1153 